\title{
Lung Field Segmentation in Digital Postero-Anterior Chest Radiographs ${ }^{\star}$
}

\author{
Paola Campadelli and Elena Casiraghi \\ Università degli Studi di Milano, \\ Computer Science Department, LAIV Laboratory \\ \{Campadelli, Casiraghi\}@dsi.unimi.it \\ http://homes.dsi.unimi.it/ campadell/LAIV
}

\begin{abstract}
This paper describes a lung field segmentation method, working on digital Postero-Anterior chest radiographs. The lung border is detected by integrating the results obtained by two simple and classical edge detectors, thus exploiting their complementary advantages. The method makes no assumption regarding the chest position, size and orientation; it has been tested on a non-trivial set of real life cases, composed of 412 radiographs belonging to two different databases. The obtained results and the comparison with more complicate techniques presented in the literature, prove the robustness of the algorithm and demonstrate that rather simple and general methods, properly combined to fit the requirements of a specific application, can provide better results.
\end{abstract}

\section{Introduction}

At the present time, chest radiography is the most common type of screening procedure for the initial detection of every type of abnormal pulmonary condition, due to its ability of revealing some unsuspected pathologic alterations, its noninvasivity characteristics, radiation dose and economic considerations. Nevertheless this is the most difficult radiograph to produce technically and to interpret diagnostically, as discussed in 1. The first and mandatory step of an automatic system aimed at any type of computerized analysis on chest radiographs, is the lung field segmentation. At the state of the art, several lung segmentation methods based on classical techniques have been presented (e.g. [2], 3], 4], [5], 6], [7] [8], 9], [10]), and reviewed in [11]. Their weakness is due to the fact that they are based on assumptions regarding the chest position, size and orientation, and often violated in real life situations. Other methods have been proposed (e.g. [12, [13]), which get better results without making these strong assumptions, but by simply including some necessary knowledge about the generic chest shape.

Considering that the loss of (even small) parts of the lung area may cause the loss of some nodules, precision is a strong requirement when the segmentation mask defines the processing area of an automatic system aimed at the detection of subtle pathologies such as lung tumors. None of the known methods obtain

\footnotetext{
* Work partially financed by CIMAINA and PRIN 2004: "Novel clustering techniques in biomedical image segmentation". 
precise results; moreover all of them detect the most visible parts of the left and right lung (visible lung areas), excluding those hidden behind the diaphragm, the heart and the spinal column (hidden lung areas), where tumors could be found.

All these reasons motivate the development of the segmentation method described in this paper, which is the first step of a lung nodules detection system. The algorithm avoids all the assumptions regarding the chest position, size and orientation, and it includes in the segmentation mask also the hidden lung areas. A precise contour is obtained by properly combining the results of two simple image segmentation techniques (sec. 22). Their appropriate integration takes into account the generic chest shape and can therefore recognize and repair their complementary errors, by exploiting the good characteristics of their individual results. For the same reason, the following step (sec. 3) separates the hidden from the visible lung areas by combining the results of simple derivative filters and those of a gray level clustering method.

The method has been developed and tested on 247 radiographs (154 of patients with lung nodules) in the standard JSRT database [14, which is the only known standard database publicly available. The images have been digitized with a $0.165 \mathrm{~mm}$ pixel size, a matrix size of $2048 \times 2048$, and 4096 gray levels. Before processing, they have been down-sampled to a dimension of $256 \times 256$ pixels (and will be referred as the Images): this experimental choice reduces the computational costs of the algorithm without worsening its performance. The algorithm has been also tested on 165 radiographs acquired from the Niguarda Hospital in Milan. They have a $0.160 \mathrm{~mm}$ pixel size, and a not fixed squared size. The comparison with the segmentation results obtained by the method described in [12], and applied to the JSRT database, proves that this is a better initialization step for a lung nodule detection system.

\section{Segmentation of the Full Lung Area}

In this section we will present the technique used for determining the external lung contour and the segmentation mask. The method that detects the external lung contour is based on the integration of the results obtained by two different algorithms. The first one detects the most visible lung edges, by means of steerable first derivative of Gaussian filters (sec. 2.1). These edges are also used to find the vertical lung axis and to initialize the second algorithm, which needs a starting point to track the lung edges and to detect a continuous contour along the external lung border (sec. 2.2). In order to define the segmentation mask, the obtained results are integrated, and other simple techniques are employed to define the bottom borders and those nearby the spine (sec. 2.3). A schematic diagram with the main steps of the method is shown in the top row of fig. 1.

\subsection{Edge Detection by Derivatives of Gaussian Filters}

The most visible lung edges are obtained by filtering the Image with first derivatives of Gaussian filters [15] at scale $\sigma=1$, oriented along four directions $(0, \pi / 4$, $\pi / 2,3 \pi / 4)$; for each direction, the top $10 \%$ of the output pixels are selected to 


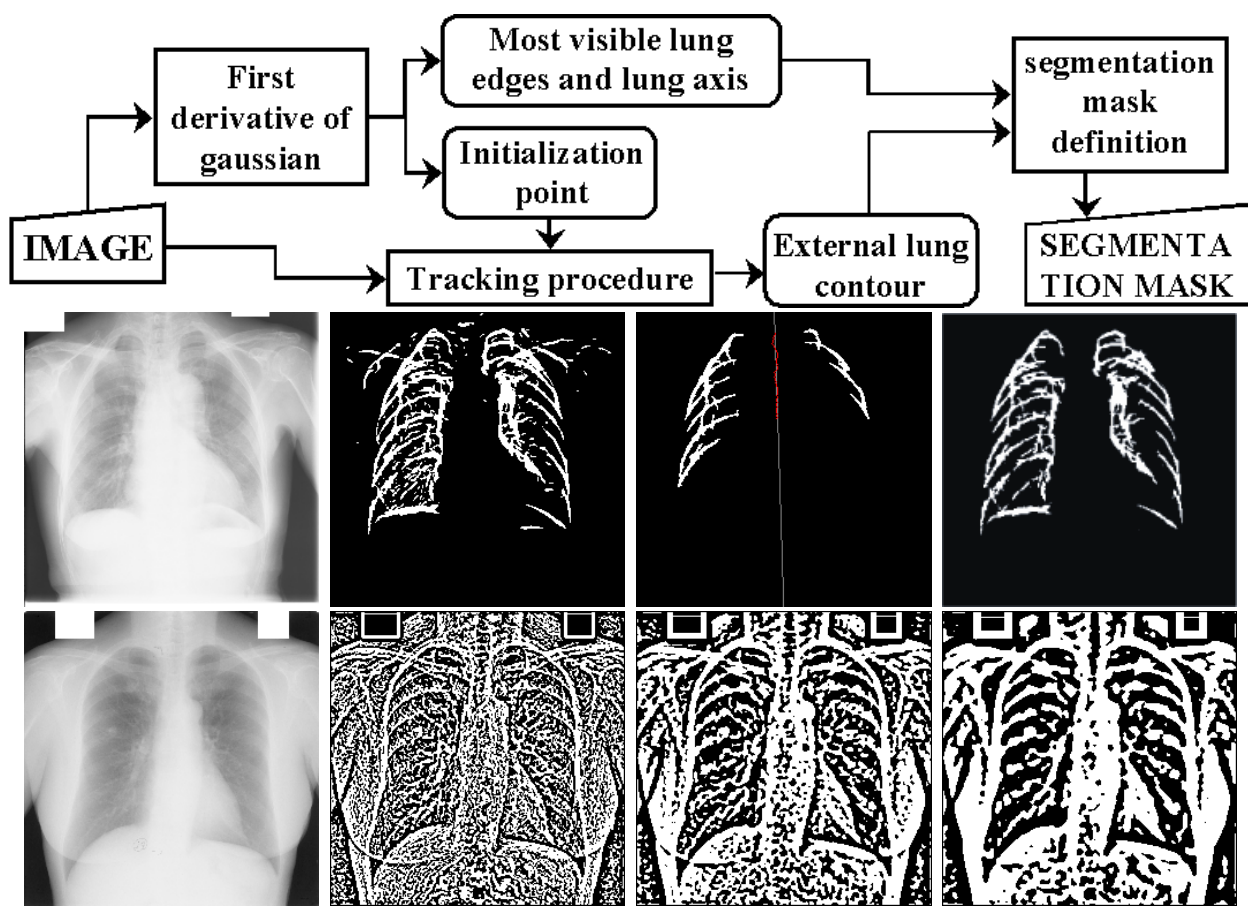

Fig. 1. Top row: Schematic diagram of the method; Middle row: Image, Initial Edge image and Partial Edge image, Edge image; Bottom row: the binary images $L_{\sigma}(\sigma=$ $1,2,3)$, capturing different levels of detail of the original image.

get four different Binary images, $B_{i}$. Their union (Initial Edge image) contains all the detected edges, while the union of $B_{\pi / 2}, B_{\pi / 4}$ and $B_{3 \pi / 4}$ (Partial Edge image) contains the main external rib cage borders and it is then used to find the vertical lung axis. To this aim each one of its rows is scanned to find the pixel at the center of the segment connecting the leftmost and rightmost pixels. A polynomial fitting method, that minimizes the $\chi$ - square error statistic, is used to fit all the found points with a line that defines the axis of the lung field (fig. 1 middle row). The axis is approximately located at the center of the spine, and it is therefore used to recognize and delete those edge regions, in the Initial Edge image, which belong to the dorsal column: they are the connected regions located in a "band" around the axis whose width is equal to $1 / 30$ of the maximum width of the chest (this estimate is given by the maximum distance between the leftmost and rightmost pixels found in each row of the Partial Edge image, and used to find the axis). Other spurious details in the Initial Edge image correspond to little regions external to the lung; they are discarded by eliminating all the connected regions whose area is less than $0.05 \%$ of the total image area. The Edge image thus obtained contains a discontinuous outline of the lung borders (fig. 1, rightmost in the middle row); to get a continuous con- 
tour a second edge detection method is presented in the next section and the two results are then integrated (sec. 2.3).

\subsection{External Contour Creation by Edge Tracking}

In this section we describe a multi-scale method to get a continuous border of the lung field. As noted in [16, "an inherent property of objects in the world is that they only exist as meaningful entities over certain ranges of scale". In the case of lung borders their analysis at a small scale provides their more accurate representation, but the segmentation could be mislead by other fine details belonging to different structures. Alternatively a large scale guarantees smoothness and continuity of the contour. For this reason we choose to process the image at different scales, and finally combine the results; the combining procedure exploits the precision of the results obtained at the smallest scale, but at the same time produces reliable results thanks to the information obtained at larger scales. Our method works on three binary images $L_{\sigma}(\sigma=1,2,3)$ each capturing different levels of detail of the lung borders (fig. 1, bottom row); each $L_{\sigma}$ is created by applying to the Image the Laplacian of Gaussian operator at scales $\sigma=1,2,3$ experimentally set, and by selecting the output pixels with positive values. An edge tracking algorithm is applied to each $L_{\sigma}$ image, in order to detect a continuous path describing the lung border at that scale; afterwards the different paths are fused to produce a robust external lung contour. The method works separately on the left and right lung; in the following we will describe it, for the generic image $L_{\sigma}$, and just for the left lung1.

The starting point, $P_{S}^{B}$, for the edge tracking procedure must belong to the external border of the left lung, and it is therefore selected from the Partial edge image; $P_{S}^{B}$ is the topmost point between those at the minimum distance from a line oriented at $3 \pi / 4$, and passing trough the origin of the coordinate system. This simple method finds a point located approximately on the top of the lateral part of the lung boundaries, where the lung border curvature changes greatly, since the edge orientation changes from horizontal (on the top border) to vertical (on the lateral border). If the pixel in position $P_{S}^{B}$ is not set to 1 in the $L_{\sigma}$ imag 2 , it is replaced by another point; the new $P_{S}^{B}$ is the first point set to 1 , and found by scanning a search area in the $L_{\sigma}$ (fig. 2, left of top row), which is a parallelogram located below the old $P_{S}^{B}$, and on its left side. If no point is found, the algorithm reaches the end without creating any contour.

The edge tracking procedure creates two continuous contours along the external border of the considered lung: the Bpath is a contour starting from $P_{S}^{B}$ and running towards the bottom of the chest, meanwhile the Tpath goes from $P_{S}^{B}$ towards the top.

\footnotetext{
${ }^{1}$ The coordinate system has the origin in the top left corner of the image, the positive $Y$ axis corresponding to the height and the positive $X$ axis to the width. The lungs are distinguished by the axis found, w.r.t. their position in the image.

${ }^{2}$ Note that this point was chosen from the Partial Edge image.
} 


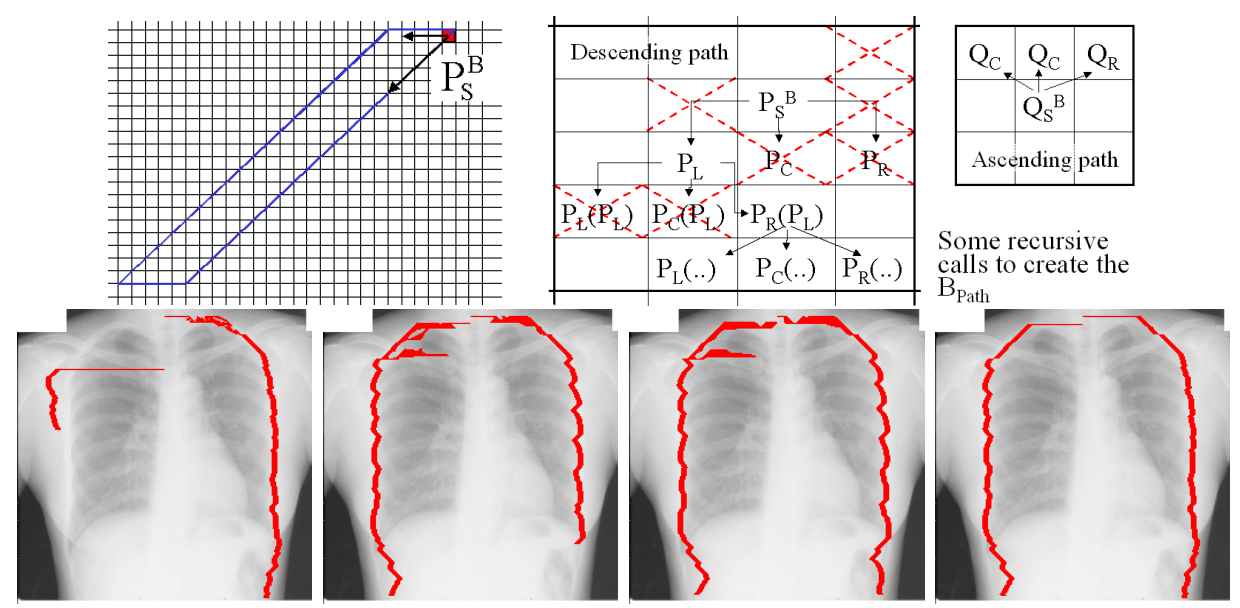

Fig. 2. top row: search area for the left path going to the bottom; Steps for the creation of the $\operatorname{DSet}_{B}\left(P_{S}^{B}\right)$ : the pixels with the cross are not inserted since set to 0 ; neighbors checked to created the $A \operatorname{Set}_{B}\left(Q_{S}^{U}\right)$; bottom row: paths created on the images $L_{\sigma}(\sigma=$ $1,2,3)$ and Border Paths created by combining the three paths.

The $\boldsymbol{B p a t h}$ is created by first defining a set of pixels, $\operatorname{DSet}_{B}\left(P_{S}^{B}\right)$, that may belong do the "descending" path. To this aim $P_{S}^{B}$ is inserted in the set and its three 8-neighbors, in the row below $\left(P_{L}, P_{C}\right.$ and $P_{R}$, see fig. (2)), are considered.

Those that are set to 1 , are inserted in the set $\operatorname{DSet}_{B}\left(P_{S}^{B}\right)$, and their three neighbors (in the row below) are recursively checked for insertion; this process goes on until at least one point is inserted in the $\operatorname{DSet}_{B}\left(P_{S}^{B}\right)$. To have a more precise contour definition, this algorithm is also run in a bottom-up direction, using as starting point, $Q_{S}^{U}$, the bottommost pixel in the $\operatorname{DSet}_{B}\left(P_{S}^{B}\right)$. Note that the neighborhood to be considered must be opportunely reversed (it is shown in fig. 22 rightmost on the top row). Once the "ascending" set $\operatorname{ASet}_{B}\left(Q_{S}^{U}\right)$ is created, a unique chain of pixels (the Bpath) is created by taking, for each row, the rightmost pixel of the intersection of the $\operatorname{DSet}_{B}\left(P_{S}^{B}\right)$ and the $A \operatorname{Set}_{B}\left(Q_{S}^{U}\right)$.

The procedure used to create the $T$ path starts from the point $P_{S}^{B}$ and applies a similar recursive algorithm, just in ascending order, to the input image; as before, its output is a set of points $\operatorname{TSet}_{T}\left(P_{S}^{B}\right)$. For each point considered, and already included in the set, the algorithm considers the pixel located above it and on its right side 3 . Both these neighbors are inserted in the $\operatorname{TSet}_{T}\left(P_{S}^{B}\right)$ if they are set to 1 , and the algorithm is recursively launched using them as starting points. The Tpath is created by taking, for each row, the rightmost among the pixels in the $\operatorname{TSet}_{T}\left(P_{S}^{B}\right)$. The topmost pixel in the Tpath delineated is often above the apex point (i.e. the real lung top). Since the apex point is located where the lung border is horizontal, this point is found in the Tpath by computing its derivative; it is the first point, $t_{\sigma}$, whose derivative value is less than a threshold,

\footnotetext{
${ }^{3}$ These directions are chosen according to the shape of the lung top borders.
} 
experimentally set to 0.05 . The Bpath and Tpath form a final contour running from the top till the bottom of the chest.

The contours created on each image $L_{\sigma}$ are shown in fig. 2. On the left, one example of a too short path is shown. Note that this is not a problem since a final contour can always be recovered by integrating the available paths. Indeed at least two paths have always been found for the 412 test images.

Since the top points $t_{\sigma}=\left(x_{t_{\sigma}}, y_{t_{\sigma}}\right)(\sigma=1,2,3)$ of the three paths may not be located at the same vertical position, the vertical coordinate of the topmost point, $Y_{T o p}$, in the final border, is set to be the median of the $y_{t_{\sigma}}$. The left contour (Left border path) is then created starting from the row indexed by $Y_{T o p}$, by selecting for each row the leftmost among all the pixels belonging to the different paths available at that row.

\subsection{Definition of the Segmentation Mask}

As noticed in sec. 2.1, even if the borders in the Edge image contain lots of edge pixels that are precisely adhering to the real lung borders, they are not continuous, the edges nearby the costophrenic angles could be missing and some edge pixels could be present that do not belong to the external borders of the lung field. On the other hand the contour obtained with the edge tracking algorithm, even though less precise, is always continuous and runs from a vertical position, that is a good approximation of the apex point of the lung, to the bottom of the imag 4 . Therefore we developed a method that composes the correct information given by the two techniques, and defines a continuous Lateral contour; in this section we describe it for the left lung.

The integration procedure starts from the topmost point in the Edge image, and below $Y_{T o p}$, and follows its external border to search for a continuous edge; when a hole is found, it is filled in by taking the pixels in the Left border path, whose advantage is to be always continuous. When the bottommost point, $L_{\text {angle }}$, in the Edge image is reached, the procedure ends after having included into the Left Lateral contour the part of Left border path below $L_{\text {angle. }}$. To define a bottom boundary for the lung field, the positions of the costophrenic angles must be defined. These points are located at the intersection of the lateral contours and the bottom edges of the lung, due to the presence of the diaphragm. These are selected from the Edge image by searching for the region, on the right side of the pixel in the position of $L_{\text {angle }}$, whose bottommost point is the nearest to $L_{\text {angle }}$ itself. The 5 leftmost and bottommost points of this region, are fitted with the method used in sec. 2.1 to find a line $l$ interpolating them. The intersection between $l$ (green colored in fig. 3) and the Lateral contour detected defines the point where the costophrenic angle is located. Repeating the same procedure for the right side the right costophrenic angle is found. A segment connecting the two costophrenic points closes the contour at the bottom. Nearby the spine, a

\footnotetext{
${ }^{4}$ Observe that the points characterizing the costophrenic angles are still uncertain since the contour, built by the edge tracking algorithm often runs till the bottom margin of the image, meanwhile the bottommost points in the left side of the Edge image may not correspond to the location of the costophrenic angle.
} 
line parallel to the lung axis is created for each lung; it passes through the point in the Edge image that is the nearest to the axis.

\section{Segmentation of the Visible Lung Area}

In this section we describe the steps that detect the contours of the spine, the diaphragm and the heart, to separate the visible from the hidden areas. Since those two areas differ mainly for their pixels gray level, the separation is performed by a contour following procedure whose result is integrated with the one produced by a gray level clustering method [17. This separation step has been helpful to detect and correct some errors of the previously described segmentation algorithm; furthermore it has been used to better characterize the candidate regions extracted by our computerized lung nodules detection system [18.

\subsection{Edges Detected with Derivative Filters}

To detect the vertical edges of the spine we filter the Image, on the left and right lung separately, with the horizontal Sobel filter, and apply a contour following procedure to the results. For each lung the starting point is selected between the $5 \%$ of the points with the highest value of the derivative and it is the nearest to the axis of the lung. The contour following procedure runs in descending and ascending direction separately. It proceeds by checking, for each row, the derivative value of the 3 points which are the 8-neighbors of the one selected at the line before, to find the one with the maximum value. It stops when the last point found is located on the border of the mask.

To detect the horizontal edges of the diaphragm, the Image is filtered, in the left and right lung separately, with a vertical Sobel filter and a similar contour following procedure is applied to the result; in the following we will describe the procedure just for the left lung. To select the starting point for the contour following procedure we consider the regions corresponding to the $5 \%$ of the pixels with the highest derivative value. Those edge regions always contain the bottom borders of the visible lung area, but may also contain some unwanted edges, belonging to the intestinal gases or other external structures. These undesired regions can be easily recognized for their shape; thus, we proceed discarding the connected regions with a convex shape, and the ones with a rectangularity 5 bigger then 0.33 . The left bottom edge of the left visible lung area is then selected, for each lung, by taking the bottommost region located at the leftmost position. The starting point for the contour following procedure is selected from the bottom edge region; it is the point with the maximum value of the derivative. The procedure runs separately towards the right till it reaches the axis; running towards the left it reaches the lateral border and eventually defines a new costophrenic angle point; this allows to correct some errors due to extra parts wrongly included by the method described in sec. 2

\footnotetext{
${ }^{5}$ The rectangularity is calculated as the fraction of the area of the region itself and
} the area of the maximum bounding box including it. 

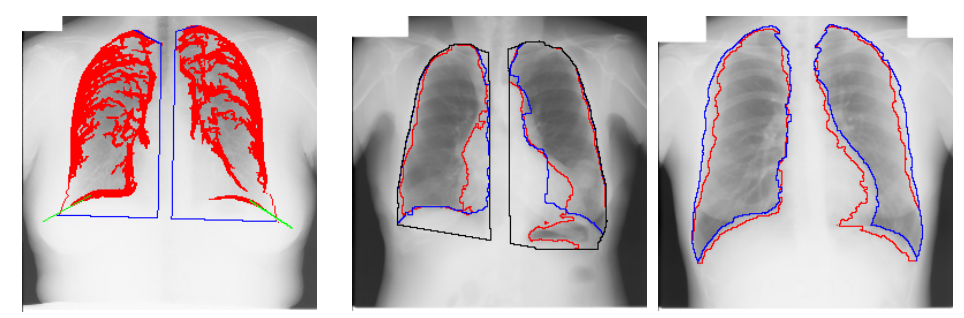

Fig. 3. Segments used to define the costophrenic angles; masks of the visible areas to be integrated.

\subsection{Clustering Method}

The second method used to define the visible areas is a clustering algorithm, which searches for three clusters in the Image. Each pixel is assigned to a certain cluster according to a distance measure based on the gray level of the pixel itself. The regions formed by the pixels belonging to the cluster whose representative has the highest gray value are then selected to give a rough definition of the hidden areas; fig. 3 shows the regions thus detected (red colored), together with the ones obtained by the derivative filters (blue colored). Note that the clustering wrongly excludes from the visible areas some regions attached to the external borders of the mask. They are ignored since we consider as external borders those created by the segmentation algorithm described in sec. 22 The two border lines that must be considered are the ones going from the top till the bottom of each lung and near to the axis; they are integrated by choosing for each row, the edge point which is the furthest from the axis.

\section{Results}

We tested our segmentation method on a rather substantial, relevant and nontrivial database of real life cases composed of the 247 images, in the JSRT database, and the 165 images, in the Niguarda database. To judge the obtained results, we compared the segmentation masks, created by our algorithm, with the true lung masks (the ground truth), manually defined by a human observer who traced the lung field borders. In this way we could detect the pixels wrongly included or excluded by each segmentation mask (the wrong pixels), and we defined as "error measure" the ratio between the number of the wrong pixels and the number of pixels in the true lung mask. This measure takes into account the big variations between the size of the chest of different patients. We detected errors when the error measure is bigger than 0.003 .

According to this performance evaluation method, all the images in the $J S R T$ database have been correctly segmented; meanwhile in the Niguarda database an error is detected in 6 images, shown in fig. 4 (i.e. the $4 \%$ of the total number of images in this database). Table 1 lists, for the 6 images, the 

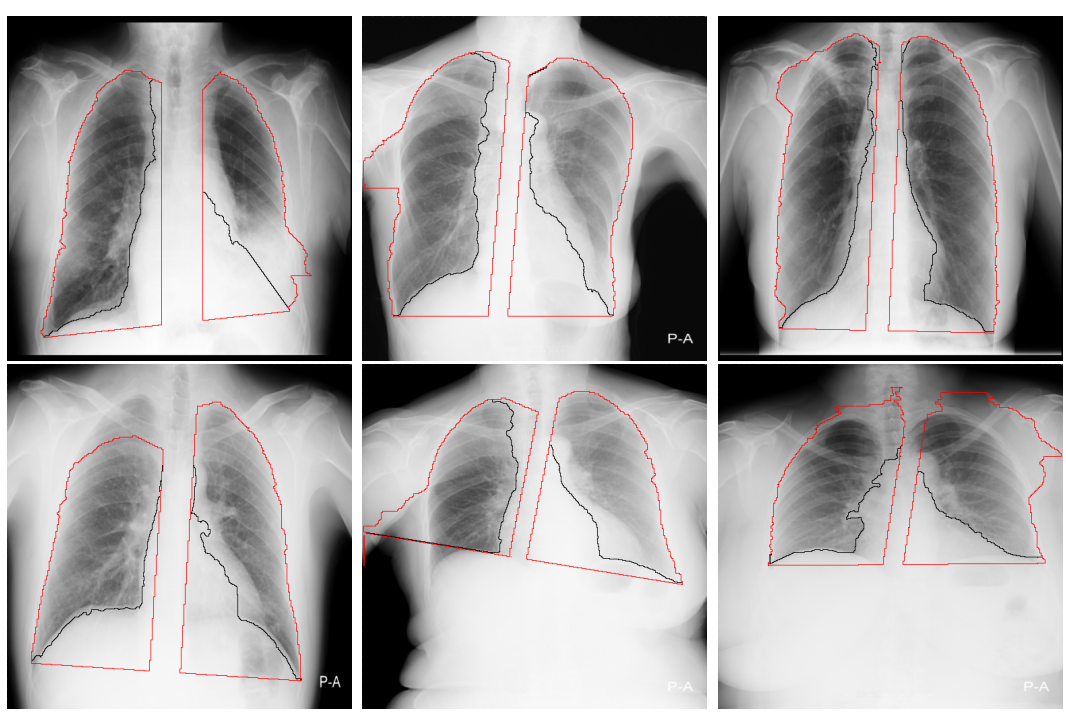

Fig. 4. results on the Niguarda (more critical) database: Top row - Image1, Image2, Image3, Bottom row - Image4, Image5, Image6.

Table 1. The errors in the six images

\begin{tabular}{|l|l|l|l|l|l|l|}
\hline & Image1 & Image2 & Image3 & Image4 & Image5 & Image6 \\
\hline \# of the wrong pixels & 368 & 897 & 2289 & 5818 & 3902 & 4100 \\
\hline True lung mask area & 25672 & 28558 & 31074 & 30107 & 18407 & 17544 \\
\hline Error measure & 0.01 & 0.03 & 0.07 & 0.19 & 0.21 & 0.23 \\
\hline
\end{tabular}

number of wrong pixels, the pixel area of the true lung mask and the error measure. Notice that this is a good performance especially because the images in the Niguarda database are of low quality: they are noisy and often characterized by low contrast. Beside, lots of them contain chests with structural abnormalities, the patients are often tilted or rotated, the position of the chest in the radiograph is not always at the center and there is a lot of variation in the size of the thoraxes. Regarding the separation between the visible and the hidden areas, it is always precise.

To have a further proof of the efficacy of our method with respect to the existing strategies, we compared it to the lung segmentation method that has been reported as one of the best performing [12, and it has been applied to the JSRT database. Since its segmentation masks do not include the hidden areas they have been extended nearby the spine and at the bottom, as described in sec. 2.3. We used the same quantitative criteria to judge those results and we found 50 images with errors, their mean error measure is 0.1 , and almost all of them are due to missing parts. Note that, if we should use this method 
to initialize a lung nodule detection system working on the $J S R T$ database, 13 nodules would be lost; on the contrary, none of them are lost by our segmentation masks, confirming that this is a good initialization step for a lung nodule detection system.

\section{References}

1. Cj Vyborny, "The aapm/rsna physics tutorial for residents: Image quality and the clinical radiographic examination.," Radiographics, vol. 17, pp. 479-498, 1997.

2. A.Hasegawa et al, "Convolution neural network based detection of lung structure," Proc. SPIE 2167, pp. 654-662, 1994.

3. J.Duryea and J.M.Boone, "A fully automatic algorithm for the segmentation of lung fields in digital chest radiographic images," Med. Phys., vol. 22, 1995.

4. X.W.Xu and K.Doi, "Image feature anlysis for computer aided diagnosis: accurate determination of ribcage boundaries chest radiographs," Med. Phys., vol. 22, 1995.

5. M.F. McNitt-Gray, H.K. Huang, and J.W. Sayre, "Feature selection in the pattern classification problem of digital chest radiographs segmentation," IEEE Trans. on Med. Imaging, vol. 14, 1995.

6. S.G.Armato et al., "Automated lung segmentation in digitized posteroanterior chest radiographs," Academic radiology, vol. 5, 1998.

7. M.S. Brown, L.S. Wilson, B.D. Doust, R.W. Gill, and C.Sun, "Knowledge-based method for segmentation and analysis of lung boundaries in chest x-rays images," Computerized Medical Imaging and Graphics, vol. 22, pp. 463-477, 1998.

8. F.M.Carrascal et al., "Automatic calculation of total lung capacity from automatically traced lung boundaries in postero-anterior and lateral digital chest radiographs," Medical Physics, vol. 25, pp. 1118-1131, 1998.

9. O. Tsuji et al., "Automated segmentation of anatomic regions in chest radiographs using an adaptive-sized hybrid neural network," Med. Phys., vol. 25, 1998.

10. N.F. Vittitoe, R. Vargas-Voracek, and C.E. Floyd Jr., "Markov random field modeling in posteroanterior chest radiograph segmentation," Med. Phys., vol. 26, 1999.

11. B. van Ginneken, B.M.ter H. Romeny, and M. Viergever, "Computer-aided diagnosis in chest radiography: A survey," IEEE Trans. On Med. Imag., vol. 20, pp. 1228-1241, 2001.

12. B.van Ginneken and B.M.ter H. Romeny, "Automatic segmentation of lung fields in chest radiographs," Medical Physics, vol. 27, 2000.

13. B.van Ginneken, "Computer-aided diagnosis in chest radiographs," P.h.D. dissertation, Utrecht Univ., Utrecht, The Nederlands, 2001.

14. Shiraishi et al, "Development of a digital image database for chest radiographs with and without a lung nodule," $A J R$, vol. 174, 2000.

15. W.T.Freeman and E.H.Adelson, "The design and use of steerable filters," IEEE Trans. on Pattern Analysis and Machine Intelligence, vol. 13, 1991.

16. T. Lindeberg, "Scale-space: A framework for handling image structures at multiple scales," Proceedings of CERN, School of Computing, vol. The Nederlands, 1996.

17. T.Uchiyama and M.Arbib, "Color image segmentation using competitive learning," IEEE Trans. on Pattern An. and Machine Int., vol. 16, 1994.

18. P.Campadelli, E.Casiraghi, and G.Valentini, "Support vector machines for candidate nodules classification," Neurocomputing (Elsevier), In Press, 2005. 